\title{
Chemical Abundances of the S Star GZ Peg
}

\author{
L. Pompéia ${ }^{\mathrm{A}}$ \\ A IP\&D, Universidade do Vale do Paraíba, Av. Shishima Hifumi, 2911, São, \\ José dos Campos, 12244-000 SP, Brazil. Email: pompeia@univap.br
}

Received 2008 November 15, accepted 2009 March 2

\begin{abstract}
The chemical compositions of stars from the Asymptotic Giant Branch are still poorly known due to the low temperatures of their atmospheres and therefore the presence of many molecular transitions hampering the analysis of atomic lines. One way to overcome this difficulty is by the study of lines in regions free from molecular contamination. We have chosen some of those regions to study the chemical abundance of the S-type star GZ Peg. Stellar parameters are derived from spectroscopic analysis and a metallicity of -0.77 dex is found. Chemical abundances of 9 elements are reported and an enhancement of $s$-process elements is inferred, typical to that of an S-type star.
\end{abstract}

Keywords: stars: abundance, stars: evolution, stars: late-type

\section{Introduction}

The Asymptotic Giant Branch (AGB) is one of the most important phases of stellar evolution due to the rich nucleosynthesis that occurs during this stage, and therefore AGB stars are crucial objects for studies of Galactic chemical evolution. During this epoch stars produce important amounts of carbons and $s$-process elements which are brought to their atmospheres by deep dredged-up events and then ejected to the interstellar medium by stellar winds. Although fundamental stones for the understanding of the final phases of low-mass stellar evolution and the stellar populations abundances, the chemical composition of such stars are still poorly known due to their low temperatures and to the blanketing of the atomic lines by molecular transitions. Some authors have tried to overcome this problem by selecting spectral regions free from molecular contaminations or the so called molecular windows (e.g. Smith \& Lambert 1985, 1990; Lambert et al. 1995; Vanture \& Wallerstein 2002a,b, 2003), and the use of the near-infrared and infrared regions (e.g. Rich \& Origlia 2005; Cunha \& Smith 2006; Woolf \& Wallerstein $2005 a, b)$. Although working with a smaller number of lines, the chemical picture of these stars can still be delineated, provided that a careful inspection of the continuum is made and an appropriate model atmosphere is adopted. The knowledge of the elemental abundances of such stars is an important tool for the understanding of the many steps followed by red giants along the $\mathrm{M}-\mathrm{MS}-\mathrm{S}-\mathrm{C}$ spectral sequence, and their role in the chemical evolution of galaxies.

In the present work we report the chemical abundances of GZ Peg, a S-type star. S stars show molecular transitions of $\mathrm{ZrO}$ and $\mathrm{LaO}$, indicating an important enrichement of $s$-process elements. Although rich in those elements, lines of the unstable $s$-element ${ }^{99} \mathrm{Tc}$ (which has a half-life of $\sim 10^{6}$ years) are not observed in GZ Peg spectrum (Lebzelter \& Hron 1999). GZ Peg is therefore classified as an extrinsic $\mathrm{S}$ star, which is predicted to have inherited its $s$-process content in a previous mass-tranfer event from an AGB compagnion. In intrinsic $\mathrm{S}$ giants, lines of Tc are observed and they are believed to have recently formed $s$-process elements and dredged-up to their atmospheres. The improvement of the quality of the stellar spectra and of the atomic constants and the availability of new model atmospheres allow now a robust analysis of the chemical patterns of such stars. This is the first of a series of papers about the detailed chemical abundance of $s$-process enriched stars. In this series the following questions are adressed: (i) What is the detailed chemical pattern of the sequence M-MS-S stars? (ii) Are there S stars with different $s$-process contamination as seen in Ba stars? And if yes, what are the factors determining the intensity of $s$-process contamination?; (iii) Does the stellar population to which the AGB star belongs affects the $s$-process production? If yes, what are the effects of this relationship?

\section{Observations and Analysis}

Observations were carried out at the 1.52-m telescope of ESO (European Southern Observatory), La Silla, in September 1999. The spectra were obtained using the FEROS spectrograph (Fiber-fed Extended Range Optical Spectrograph) with wavelength range 356 to $920 \mathrm{~nm}$ and a resolution of $R=48000$. Reductions were performed using the DRS (online data reduction system of FEROS). A subsequent reduction was performed using the CONTINUUM, RVIDLINES and DOPCOR tasks of the IRAF package. The mean signal to noisy ratio of the spectrum is $S / N=61.97$. 


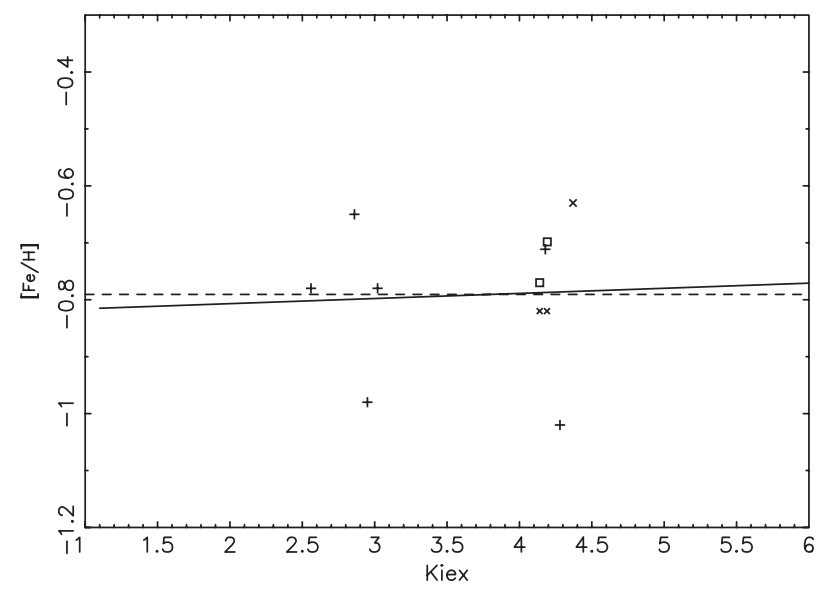

Figure 1 Excitation equilibrium of the Fe I lines for GZ Peg.

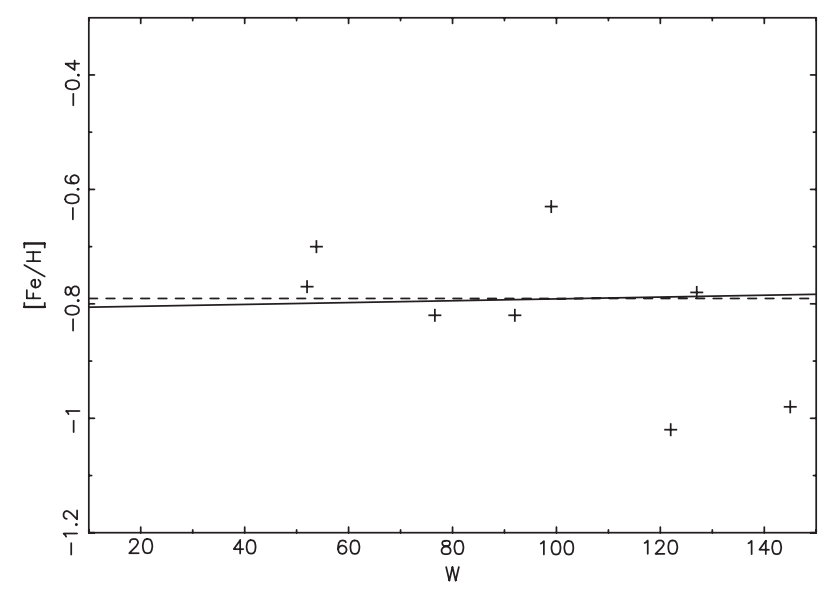

Figure $2[\mathrm{Fe} / \mathrm{H}]$ versus $E W$ of the Fe I lines for GZ Peg.

Reddening has been estimated according to Chen et al. (1998) and bolometric correction has been interpolated from the grids of Lejeune et al. (1997). GZ Peg is a semiregular (SRA) variable according to the General Catalog of Variable stars (GCVS, Samus et al. 2004). The light curve of the star at AAVSO (American Association of Variable Star Observers) has been checked and a visual magnitude range of $\pm 0.4 \mathrm{mag}$ is found for this star, therefore an uncertainty of $0.4 \mathrm{mag}$ is expected. We have adopted the bolometric luminosity of $L_{\text {bol }}=-5.02$ from Guandalini \& Busso (2008). The temperature was inferred by the IRFM using the empirical calibrations of Alonso, Arribas \& Martínez-Roger (1999) and 2MASS colors. The resulting values are $T(V-K)=3502 \mathrm{~K}, T(J-H)=3621 \mathrm{~K}$ and $T(J-K)=3454 \mathrm{~K}$. These temperatures have been checked by the excitation equilibrium of the Fe I lines (Figure 1), and a best fit has been found for $T_{\text {eff }}=3600 \mathrm{~K}$. Although $S$ stars are predicted to have masses in a range of 1.5-2 $\mathrm{M}_{\odot}$ (Jorissen et al. 1998), GZ Peg is an extrinsic $\mathrm{S}$ star, therefore a series of tests have been made to better constrain the mass of this star. Tests requiring simultaneously (i) the excitation equilibrium of the Fe I lines and (ii) the curve of growth fitting, were made with masses of

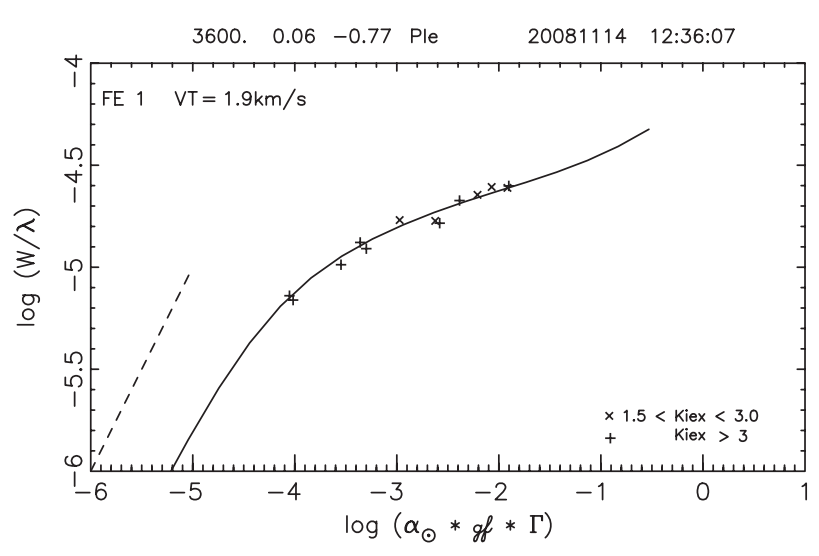

Figure 3 Curve of growth fitting.

Table 1. Changes in chemical abundances due to stellarparameter uncertainties

\begin{tabular}{lcrccc}
\hline Element & $\begin{array}{c}\Delta \log g \\
+0.2 \mathrm{dex}\end{array}$ & $\begin{array}{c}\Delta T_{\text {eff }}+50 \mathrm{~K} \\
+[\mathrm{Fe} / \mathrm{H}] \\
+0.15 \mathrm{dex}\end{array}$ & $\begin{array}{c}\Delta \xi \\
0.10 \mathrm{~km} \mathrm{~s}^{-1}\end{array}$ & $\begin{array}{c}\text { Total error } \\
\delta\end{array}$ \\
\hline Fe 1 & 0.09 & -0.03 & -0.01 & -0.05 & 0.11 \\
Ti 1 & 0.05 & 0.04 & -0.03 & 0.00 & 0.10 \\
$\mathrm{Ni} \mathrm{1}$ & 0.07 & -0.05 & -0.03 & 0.00 & 0.09 \\
$\mathrm{Cr} 1$ & 0.04 & 0.02 & -0.01 & 0.00 & 0.07 \\
$\mathrm{Zr} \mathrm{1}$ & 0.07 & 0.07 & -0.02 & 0.00 & 0.11 \\
$\mathrm{Nd} \mathrm{2}$ & 0.08 & -0.02 & 0.08 & 0.00 & 0.11 \\
Y 2 & 0.09 & -0.02 & -0.05 & 0.00 & 0.10 \\
$\mathrm{Ce} 2$ & 0.09 & 0.01 & 0.05 & 0.00 & 0.10 \\
Ba 1 & 0.02 & 0.05 & 0.01 & 0.00 & 0.05 \\
\hline
\end{tabular}

Table 2. Chemical abundances

\begin{tabular}{lrcc}
\hline Element & {$[\mathrm{X} / \mathrm{H}]$} & $\delta^{\mathrm{a}}$ & Number of lines \\
\hline Fe 1 & -0.77 & 0.04 & 13 \\
Ti 1 & -0.42 & 0.11 & 9 \\
Ni 1 & -0.62 & 0.13 & 6 \\
Zr 1 & -0.19 & 0.11 & 11 \\
Cr 1 & -0.28 & 0.32 & 2 \\
Nd 2 & 0.35 & 0.13 & 5 \\
Y 2 & -0.10 & - & 1 \\
Ce 2 & 0.56 & 0.27 & 2 \\
Ba 1 & -0.05 & - & 1 \\
\hline
\end{tabular}

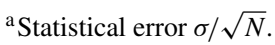

$3 \mathrm{M}_{\odot}$ to $1.5 \mathrm{M}_{\odot}$ and the iteration procedure converged for a model with $2 \mathrm{M}_{\odot}$. This value has been adopted to derive the trigonometric surface gravity also using the Hipparcos paralaxe.

The stellar parameters have been inferred by requiring the ionization equilibrium of the Fe I and Fe II lines to derive the spectroscopic gravity and metallicity, and zero slope in the $[\mathrm{Fe} / \mathrm{H}]$ versus $E W$ (equivalent width) plot to infer the microturbulence velocity. The resulting stellar parameters are the following: $T_{\text {eff }}=3600 \mathrm{~K}, \log g=0.06$, 
Table 3. Line list

\begin{tabular}{|c|c|c|c|c|c|}
\hline Wavelength & Element & $\chi_{\text {exc }}$ & $\log g f$ & Reference & $E W$ \\
\hline 7411.180 & FE1 & 4.283 & -0.299 & 02 & 122.0 \\
\hline 7418.670 & FE1 & 4.143 & -1.226 & 02 & 92.0 \\
\hline 7443.002 & FE1 & 4.186 & -1.405 & 02 & 76.6 \\
\hline 7453.997 & FE1 & 4.186 & -1.907 & 02 & 53.8 \\
\hline 7461.530 & FE1 & 2.559 & -3.281 & 02 & 127.0 \\
\hline 7498.530 & FE1 & 4.143 & -1.940 & 02 & 52.0 \\
\hline 7511.040 & FE1 & 4.178 & 0.229 & 02 & 188.0 \\
\hline 7531.170 & FE1 & 4.371 & -0.939 & 02 & 99.0 \\
\hline 7583.800 & FE1 & 3.018 & -1.990 & 02 & 161.0 \\
\hline 8611.800 & FE1 & 2.850 & -1.926 & 01 & 213.0 \\
\hline 8621.600 & FE1 & 2.950 & -2.321 & 01 & 145.0 \\
\hline 8674.750 & FE1 & 2.830 & -1.800 & 01 & 213.0 \\
\hline 8838.430 & FE1 & 2.860 & -2.050 & 01 & 200.0 \\
\hline 7337.780 & TI1 & 2.239 & -1.517 & 02 & 119.6 \\
\hline 7474.940 & TI1 & 1.749 & -2.187 & 02 & 102.6 \\
\hline 7496.120 & TI1 & 2.236 & -0.937 & 02 & 123.6 \\
\hline 8024.840 & TI1 & 1.880 & -1.140 & 01 & 159.0 \\
\hline 8334.390 & TI1 & 0.820 & -2.637 & 01 & 206.0 \\
\hline 8364.240 & TI1 & 0.840 & -1.756 & 01 & 319.2 \\
\hline 8377.860 & TI1 & 0.830 & -1.612 & 01 & 392.6 \\
\hline 8396.900 & TI1 & 0.810 & -1.779 & 01 & 380.0 \\
\hline 8412.360 & TI1 & 0.820 & -1.483 & 01 & 388.6 \\
\hline 8416.950 & TI1 & 2.240 & -1.034 & 01 & 133.7 \\
\hline 8417.470 & TI1 & 2.120 & -1.951 & 01 & 124.8 \\
\hline 8426.510 & TI1 & 0.830 & -1.253 & 01 & 468.0 \\
\hline 8438.920 & TI1 & 2.260 & -0.800 & 01 & 153.7 \\
\hline 8450.890 & TI1 & 2.250 & -0.903 & 01 & 106.8 \\
\hline 4385.240 & NI1 & 2.740 & -2.037 & 02 & 97.3 \\
\hline 7414.500 & NI1 & 1.986 & -2.549 & 02 & 182.7 \\
\hline 7422.300 & NI1 & 3.635 & -0.251 & 02 & 133.7 \\
\hline 7525.140 & NI1 & 3.635 & -0.546 & 02 & 91.6 \\
\hline 7555.600 & NI1 & 3.847 & -0.046 & 02 & 107.4 \\
\hline 7574.043 & NI1 & 3.833 & -0.533 & 02 & 104.3 \\
\hline 7437.138 & $\mathrm{CO} 1$ & 1.956 & -2.876 & 02 & 64.6 \\
\hline 7553.963 & $\mathrm{CO} 1$ & 3.952 & -0.955 & 02 & 43.1 \\
\hline 7355.935 & CR1 & 2.890 & -0.290 & 01 & 208.0 \\
\hline 7400.226 & CR1 & 2.900 & -0.110 & 01 & 202.2 \\
\hline 7462.364 & CR1 & 2.910 & -0.040 & 01 & 213.6 \\
\hline 8348.283 & CR1 & 2.710 & -1.830 & 01 & 112.0 \\
\hline 8976.880 & CR1 & 3.090 & -1.110 & 01 & 156.2 \\
\hline 7439.890 & ZR1 & 0.543 & -1.810 & 02 & 92.9 \\
\hline 7479.532 & ZR1 & 1.834 & -1.380 & 02 & 24.8 \\
\hline 7551.493 & ZR1 & 1.584 & -1.360 & 02 & 36.9 \\
\hline 7554.780 & ZR1 & 0.520 & -2.280 & 02 & 111.6 \\
\hline 7562.129 & ZR1 & 0.623 & -2.710 & 02 & 46.9 \\
\hline 8070.115 & ZR1 & 0.730 & -0.790 & 01 & 141.1 \\
\hline 8133.011 & ZR1 & 0.690 & -1.130 & 01 & 178.8 \\
\hline 8212.577 & ZR1 & 0.650 & -1.320 & 01 & 167.2 \\
\hline 8305.987 & ZR1 & 0.620 & -1.660 & 01 & 131.4 \\
\hline 8389.491 & ZR1 & 0.600 & -1.760 & 01 & 148.7 \\
\hline 8584.281 & ZR1 & 1.875 & -1.320 & 02 & 44.2 \\
\hline 7427.416 & ND2 & 1.490 & -1.380 & 02 & 45.0 \\
\hline 7481.286 & ND2 & 0.205 & -2.900 & 02 & 30.8 \\
\hline 7577.496 & ND2 & 0.205 & -2.930 & 02 & 54.0 \\
\hline 8594.883 & ND2 & 1.140 & -1.860 & 02 & 33.9 \\
\hline 8643.478 & ND2 & 1.200 & -1.750 & 02 & 42.3 \\
\hline 7947.597 & RB1 & 0.000 & -0.170 & 01 & 104.6 \\
\hline 7450.276 & $\mathrm{Y} 2$ & 1.748 & -1.590 & 02 & 43.8 \\
\hline 8810.854 & CE1 & 0.302 & -1.665 & 02 & 18.4 \\
\hline 7486.557 & CE2 & 2.548 & -0.394 & 02 & 23.5 \\
\hline 8772.135 & CE2 & 0.357 & -2.515 & 02 & 75.4 \\
\hline 7488.077 & BA1 & 1.190 & -0.230 & 03 & 29.3 \\
\hline
\end{tabular}

Atomic References: 01, Woolf \& Wallerstein (2005b); 02, VALD ${ }^{1}$; 03, Vanture \& Wallerstein (2003).

${ }^{1}$ http://ams .astro.univie.ac.at/ $\sim$ vald/.
$[\mathrm{Fe} / \mathrm{H}]=-0.77 \mathrm{dex}$ and $\xi=1.88 \mathrm{~km} \mathrm{~s}^{-1}$. The $[\mathrm{Fe} \mathrm{I} / \mathrm{H}]$ versus $E W$ relation is depicted in Figure 2 and the curve of growth fitting is given in Figure 3. The model atmospheres adopted here is a new version of the MARCS models atmospheres (Gustafsson et al. 2008). We have selected $2 \mathrm{M}_{\odot}$ spherical models and interpolated between grids to obtain the best result.

A change of $50 \mathrm{~K}$ in the temperature yields a change in gravity of 0.025 dex and a change of 0.4 mag in the bolometric magnitude yields a change in gravity of 0.16 dex. We have determined the errors in stellar abundances due to stellar parameters uncertainties taking into account a change of $\Delta \log g=+0.2$ dex in gravity, $\Delta T_{\text {eff }}=+50 \mathrm{~K}$ in temperature, $\Delta[\mathrm{Fe} / \mathrm{H}]=+0.15$ dex in metallicity and $\Delta \xi=0.10 \mathrm{~km} \mathrm{~s}^{-1}$ in microturbulence velocity. The resulting errors are given in Table 1. The uncertainties have been added in quadrature and the total errors are given in the last column.

Chemical abundances have been inferred by the analysis of the equivalent widths of the lines. The line list and the $E W$ of the lines are given in Table 3, which gives the central wavelength of the lines, chemical element, excitation potential, oscilator strength, atomic data reference and equivalent width. In Table 2 we give the final abundances with the number of lines used in the analysis and the statistical errors $(\sigma / \sqrt{N})$.

\section{Discussion}

A somewhat low metallicity of $[\mathrm{Fe} / \mathrm{H}]=-0.77$ has been found for GZ Peg. Abundances of $[\mathrm{Ti} / \mathrm{Fe}]=+0.35$ and $[\mathrm{Ni} / \mathrm{Fe}]=+0.15$ are derived, and a slightly high value for $\mathrm{Cr},[\mathrm{Cr} / \mathrm{Fe}]=+0.48$ is found (although this element is represented only by two lines with a high scater between them). For the $s$-process elements, we have $\mathrm{Zr} \mathrm{I}$ and Nd II abundances better defined (with a larger number of lines) than Y II, Ba I and Ce II. We have found $s$-process abundances typical of a S star for GZ Peg. In Figure 4 we plot our data with those of two $\mathrm{S}$ stars from Vanture \& Wallerstein (2003), MS and S stars from Smith \& Lambert (1986) and Ba stars of Smiljanic et al. (2007) and Allen \& Barbuy (2006). As can be seen from this figure, GZ Peg shows supersolar abundance ratios for $[\mathrm{Ba} / \mathrm{Fe}],[\mathrm{Zr} / \mathrm{Fe}]$, $[\mathrm{Y} / \mathrm{Fe}],[\mathrm{Nd} / \mathrm{Fe}]$ and $[\mathrm{Ce} / \mathrm{Fe}]$, and an enhanced pattern for $[\mathrm{Nd} / \mathrm{Fe}]$ and $[\mathrm{Ce} / \mathrm{Fe}]$ when compared to most of the senriched stars. The overabundance level is similar to that of the intrinsic S star S Uma (Vanture \& Wallerstein 2003), which has a metallicity of $[\mathrm{Fe} / \mathrm{H}]=-0.3$.

In order to better understand the origin of the abundance pattern of GZ Peg, we have compared in Figure 5 the resulting abundances with those predicted by Goriely \& Mowlavi (2000) for an AGB star with metallicity $[\mathrm{Fe} / \mathrm{H}]=-0.70$ and mass of $2.5 \mathrm{M}_{\odot}$ which has undergone 10, 30 and 50 dredge-ups. Surface abundances predicted for 10 dredge-ups are depicted by the solid line, for 30 dredge-ups by the dotted line, and for 50 dredge-ups by the dashed line. Except for the Ba abundance, which has been derived from only one line, GZ Peg data are better 


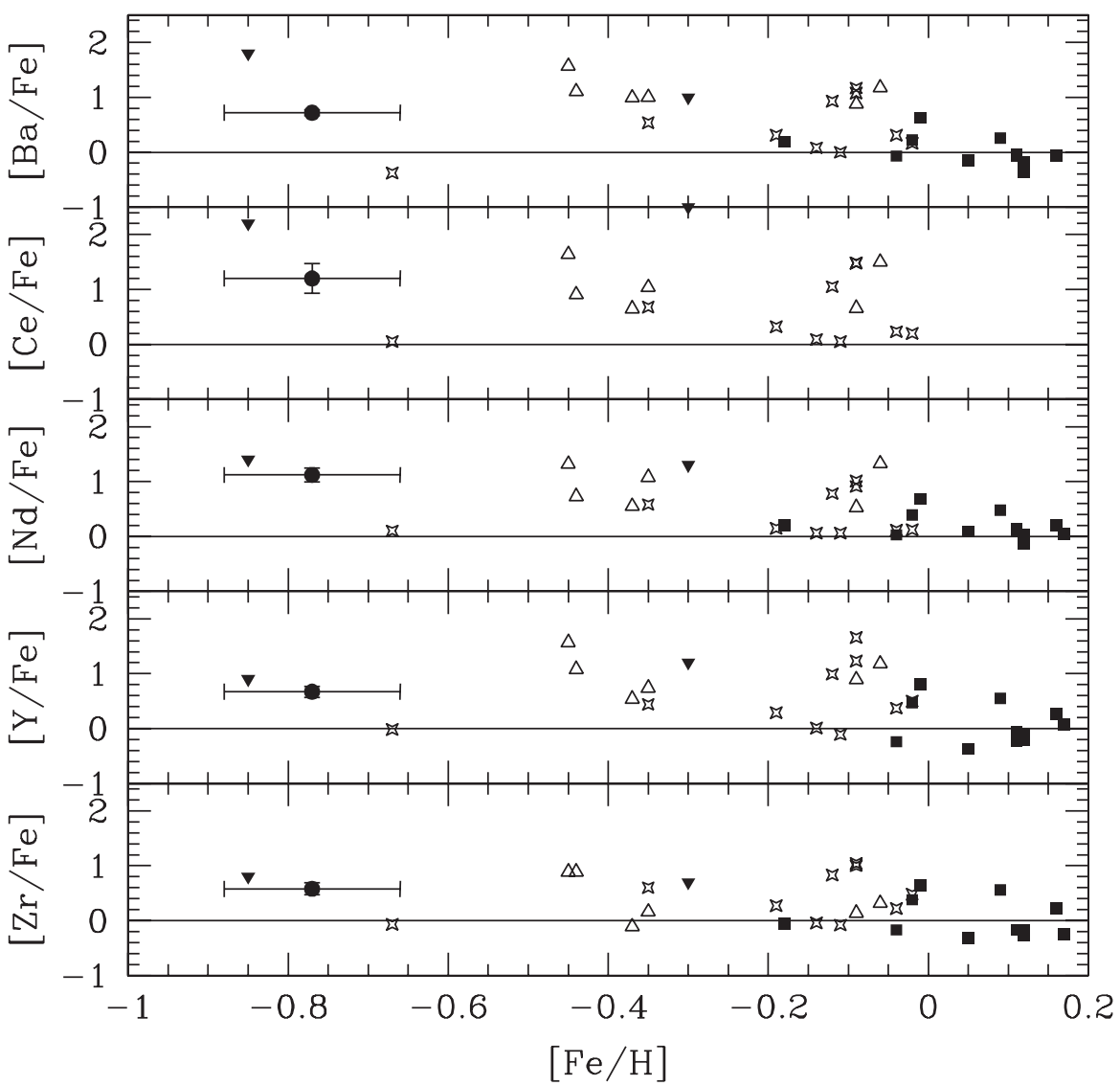

Figure 4 Comparison of the chemical abundances of GZ Peg with MS, S and Ba stars. The reference of the symbols are the following: filled circle, GZ Peg; downward-pointing triangles, S stars, from Vanture \& Wallerstein (2003); open stars, MS and S stars, from Smith \& Lambert (1986); squares, Ba stars, from Smiljanic, Porto de Mello \& da Silva (2007). For the error bars we have adopted the highest error value between Tables 2 and 1.

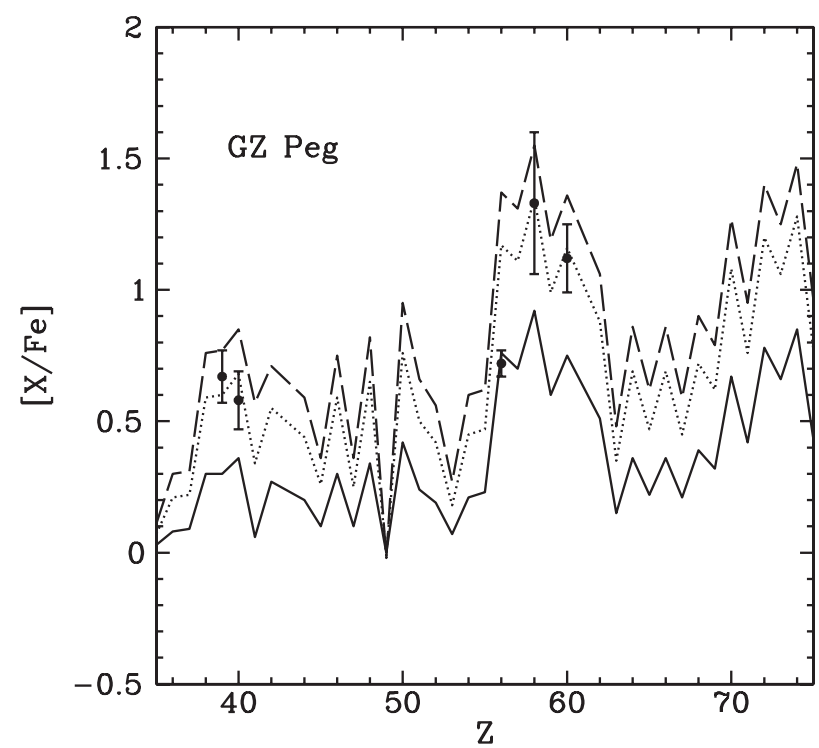

Figure $5 \quad[\mathrm{X} / \mathrm{Fe}]$ versus $\mathrm{Z}$ for GZ Peg compared to theoretical surface abundances predicted for AGB stars of Goriely \& Mowlavi (2000). Our data are compared to models with $[\mathrm{Fe} / \mathrm{H}]=-0.70$ and $M=2.5 \mathrm{M}_{\odot}$. The solid line represents surface abundances predicted for 10 dredge-ups, the dotted line for 30 dredge-ups, and the dashed line for 50 dredge-ups. Error bars are the same as in Figure 4. described by the 30-dredge-up model. Such analysis may indicate that this star has probably accreted matter from an AGB star which has passed through an important number of dredge-up events.

\section{Conclusions}

A detailed analysis of the extrinsic S star GZ Peg has been performed. The spectroscopic stellar parameters derived for this star are: $T_{\text {eff }}=3600 \mathrm{~K}, \log g=0.06$, $[\mathrm{Fe} / \mathrm{H}]=-0.77 \mathrm{dex}$ and $\xi=1.88 \mathrm{~km} \mathrm{~s}^{-1}$. The derived $s$-process abundances are in agreement with those of S-type stars or other stars with enhanced $s$-process abundances. Comparing the abundance level of GZ Peg with theoretical surface abundances of AGB stars from Goriely \& Mowlavi (2000) we have found a good agreement with the 30-dredge-up model. Such analysis indicates that the pristine AGB star which contaminated GZ Peg has passed through a considerable number of dredge-up events.

\section{Acknowledgments}

This work has made use of the SIMBAD, VALD and AAVSO databases. 


\section{References}

Allen, D. M. \& Barbuy, B., 2006, A\&A, 454, 895

Alonso, A., Arribas, S. \& Martínez-Roger, C., 1999, A\&AS, 140, 261

Cunha, K. \& Smith, V. V., 2006, ApJ, 651, 491

Goriely, S. \& Mowlavi, N., 2000, A\&A, 362, 599

Gustafsson, B., Edvardsson, B., Eriksson, K., Jørgensen, U. G., Nordlund, A. \& Plez, B., 2008, A\&A, 486, 951

Jorissen, A., Van Eck, S., Mayor, M. \& Udry, S., 1998, A\&A, 332, 877

Lebzelter, Th. \& Hron, J., 1999, A\&A, 351, 533
Rich, R. M. \& Origlia, L., 2005, ApJ, 634, 1293

Smiljanic, R., Porto de Mello, G. F. \& da Silva, L., 2007, A\&A, 468, 679

Smith, V. \& Lambert, D. L., 1985, ApJ, 294, 326

Smith, V. \& Lambert, D. L., 1986, ApJ, 311, 843

Smith, V. \& Lambert, D. L., 1990, ApJS, 72, 387

Vanture, A. \& Wallerstein, G., 2002, ApJ, 564, 395

Vanture, A. \& Wallerstein, G., 2002, ApJ, 569, 984

Vanture, A. \& Wallerstein, G., 2003, PASP, 115, 814

Woolf, V. \& Wallerstein, G., 2005a, ASPC, 336, 375

Woolf, V. \& Wallerstein, G., 2005b, MNRAS, 356, 963 